\title{
Voltammetry at electrodes decorated with an insulating porous film: Understanding the effects of adsorption
}

\author{
Henry T. H. Chan, Enno Kätelhön, and Richard G. Compton* \\ Department of Chemistry, Physical and Theoretical Chemistry Laboratory, Oxford University \\ South Parks Road, Oxford, OX1 3QZ, United Kingdom
}

\begin{abstract}
Recent literature has evinced a keen interest in porous electrodes. Numerous applications have been discussed and some aspects of the voltammetry investigated, a theoretical description of adsorptionand desorption processes though remains largely absent. The present study fills this important gap and provides a detailed analysis of the impact of adsorption on voltammetric electrode responses in porous materials. It is shown that in some cases, adsorption- and desorption processes may aid the detection of analytes while in other cases a planar unmodified electrode is superior in performance. Finally practical conclusions are drawn for the design of future experimental systems.
\end{abstract}

\section{Highlights}

- Cyclic voltammetry is investigated at adsorbing porous electrodes.

- Ad- and desorption processes may lead to amplified signals.

- Practical advice is given to experimentalists.

\section{Keywords}

Porous electrodes, adsorption processes, nanoelectrochemistry. 


\section{Introduction}

Recent years have seen a broad interest in porous electrodes ${ }^{1-8}$ in electrochemistry that is due to advantages offered in various applications: The vast increase in surface area drastically advances the electrode capacitance and hence the performance of supercapacitors, porous layers are often easier to functionalise in biosensing- and biological studies than their planar equivalents, and thin-layer diffusion effects may be exploited in electroanalytical measurements to just name a few.

The greater surface area of porous electrodes may however lead to significant adsorption of reactants in electrochemical sensing, which certainly affects the outcome of the measurement. This effect may be exploited straightforwardly at fully electrcchemically-active electrodes in anodic stripping experiments but may also be highly beneficial to electrodes decorated with a porous electrochemically-inactive film, which we address in the following. Many chemical sensors are deliberately designed to incorporate an electrochemically-inactive porous layer on the surface of an electrode in which analyte pre-concentration is used to amplify the signals recorded amperometrically. This work investigates the extent to which such adsorption effects can be helpful in such sensing applications and, where yes, how they can be exploited. To this end, we develop a simple representative model and conduct a detailed parameter study of experimentally-relevant parameter ranges. Our results reveal direct implications for experimental studies and provide useful guidance to experimentalists.

\section{Theory}

The following theory section first details the investigated theoretical model before we proceed with a short description of employed numerical methods and finally define a set of dimensionless quantities which are subsequently employed throughout most of the results and discussion in Section 3.

\subsection{Theoretical model}

We investigate porous electrodes that comprise or are approximated by a large number of vertically aligned cylindrical fibres mounted perpendicularly on a supporting surface, see Figure 1. The fibres are electrochemically inactive though adsorption and desorption processes may take place at their surfaces. Electrochemical reactions are assumed to occur solely at the supporting surface. The chosen model does not resemble a particular experimental scenario, but is intended as a general and representative system for porous electrodes and renders a sensible starting point for a first investigations. While a carbon nanotube 'forest' is roughly approximated, drawn conclusions can nonetheless be equally applied to similar porous materials including electrodes featuring holes rather than fibres or randomly-patterned porous structures.

As the mathematical description of the entire electrode structure is computationally inefficient and not of particular interest in the context of this work, we make use of the diffusion domain approximation, which has become a standard tool in the modelling of textured macroelectrodes and has previously been described in textbooks ${ }^{9 ; 10}$ and numerous publications ${ }^{11-16}$. In short, we exploit the symmetry of the electrode acknowledging the fact that in the case of regularly patterned fibres the diffusion field features a mirror symmetry with respect to Voronoi surfaces of the individual cylinder's symmetry axis. As part of the diffusion domain approximation, we can further approximate the diffusion fields adjacent to each cylinder to feature rotational symmetry with respect to the cylinders' symmetry axis. By this means the 


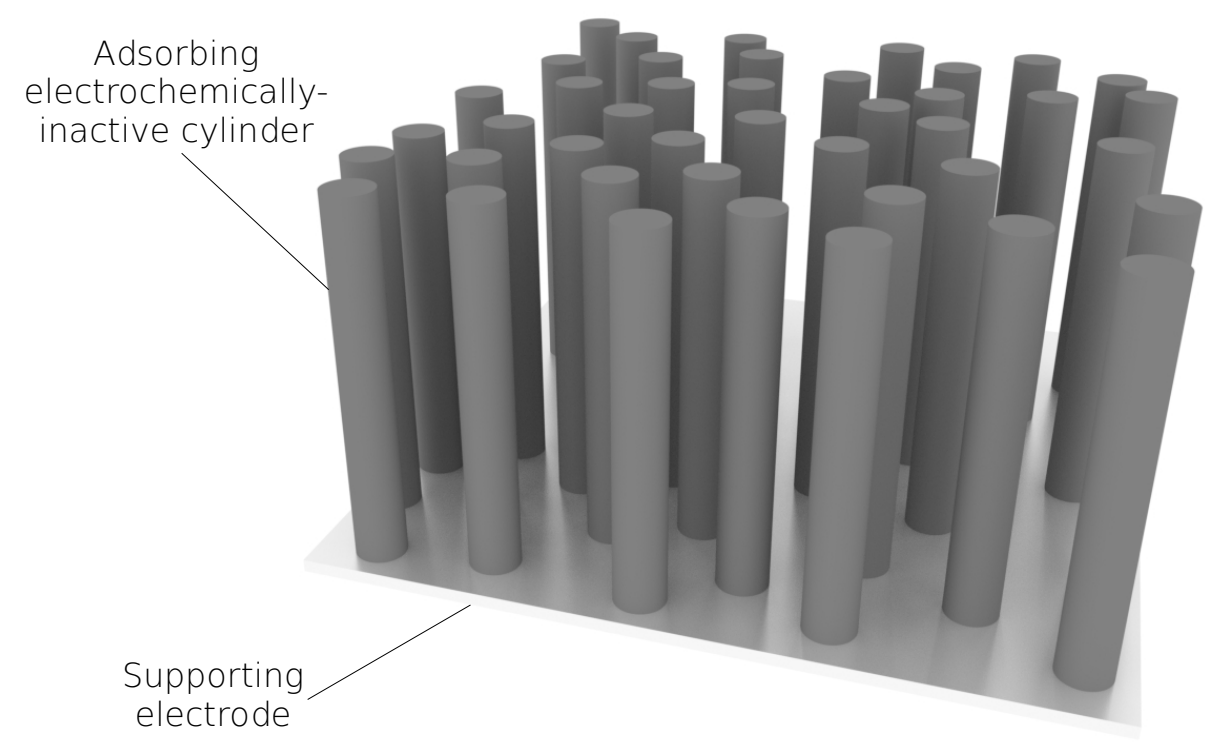

Figure 1: Illustration of the investigated electrode geometry.

geometry of the entire electrode can be simplified to a large number of identical two-dimensional cells as shown in Figure 2, where they are depicted alongside various equations we explain in detail below.

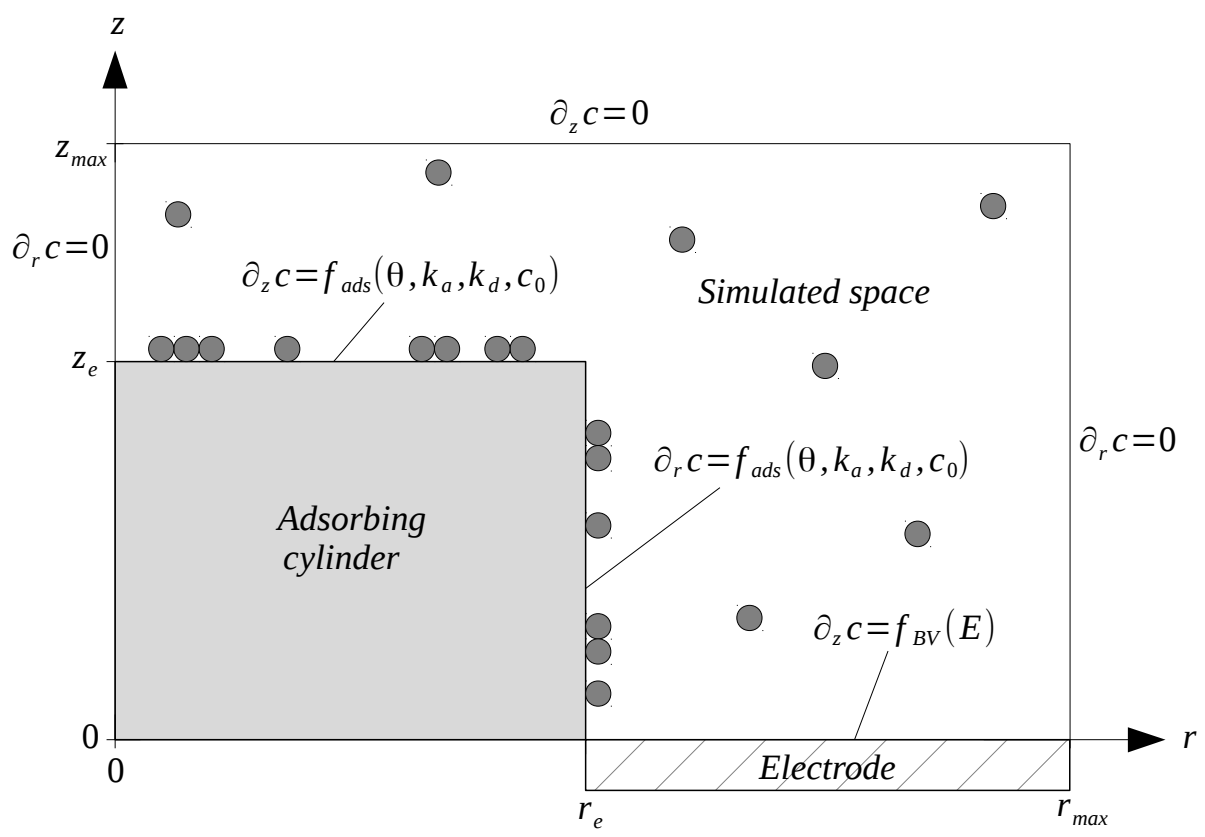

Figure 2: Illustration of the simulated geometry and the implemented boundary conditions to diffusion equation after the application of the diffusion domain approximation. Grey spheres indicate analyte molecules being either adsorbed in a monolayer on the cylinder surfaces or freely diffusing in solution. [Figure was amended.]

In our model mass transport is assumed to be of a solely diffusional nature, which is a good ap- 
proximation for most nano- and micro-scaled systems and typically holds for the time scale of standard experiments, where the influence of temperature fluctuations and related thermal convection ${ }^{17}$ can be neglected and a sufficient amount of electrolyte ${ }^{18}$ is present to avoid migration effects. The mass transport of electro-active species can hence be described as:

$$
\partial_{t} c(\vec{r}, t)=D \Delta c(\vec{r}, t)
$$

where $c$ is the concentration of the diffusing electro-active species at the position $\vec{r}$ and the time $t, D$ the diffusion coefficient, and $\Delta$ the Poisson operator.

The diffusion equation is solved subject to boundary conditions that partly arise from the combination of the diffusion domain approximation and partly from the physical properties of the system. Specific to the diffusion domain approximation are the boundary conditions set at $r=0$ and $r=r_{\max }$ which implement the system's mirror symmetry with respect to the cylinder's symmetry axis and the border of the diffusion domain discussed above. Mathematically these can be expressed as:

$$
\left.\partial_{r} c(\vec{r}, t)\right|_{r=0}=0
$$

and:

$$
\left.\partial_{r} c(\vec{r}, t)\right|_{r=r_{\max }}=0
$$

In addition we implement an upper boundary $z_{\max }$ in $z$-direction, which resembles the transition between the greatest possible diffusion field of the electrode and the bulk solution. This boundary is set in a distance far enough ${ }^{9}$ from the electrode to ensure that the electrode's diffusion field never expands to the boundary and the boundary's influence on the outcome of the simulation is negligible:

$$
\left.\partial_{z} c(\vec{r}, t)\right|_{z=z_{\max }}=0 \quad \text { with } \quad z_{\max }=6 \sqrt{D t_{\max }}+z_{e}
$$

where $t_{\max }$ is the duration of the experiment and $z_{e}$ the height of the cylinder. The physical properties of the electrode are implemented via boundary conditions that describe interface processes at the supporting electrode and the adsorbing porous structure. For the electrode we consider a simple one-electron heterogeneous reaction of the two species $A$ and $B$ :

$$
A+e \rightleftharpoons B
$$

which feature the same diffusion coefficient and hence, in the absence of or in combination with suitable boundary conditions, fulfil:

$$
c_{A}(\vec{r}, t)+c_{B}(\vec{r}, t)=c_{A}(\vec{r}, t=0)+c_{B}(\vec{r}, t=0)=c_{B}^{*}
$$

at any time, where $c_{B}^{*}$ is the initial homogeneous concentration of $B$ and the initial concentration of $A$ is set to zero. We further assume Butler-Volmer kinetics, which given the above equation can be expressed as: 


$$
\begin{aligned}
\left.D \partial_{z} c_{A}(\vec{r}, t)\right|_{r_{e}<r<r_{\max }, z=0} & =-\left.D \partial_{z} c_{B}(\vec{r}, t)\right|_{r_{e}<r<r_{\max }, z=0} \\
= & k_{0} \exp \left(\frac{-\alpha F\left(E-E_{f}^{0}\right)}{R_{u} T}\left(c_{B}^{*}-c_{B}(\vec{r}, t)\right)\right) \\
& \quad-k_{0} \exp \left(\frac{\beta F\left(E-E_{f}^{0}\right)}{R_{u} T} c_{B}(\vec{r}, t)\right) \\
& =f_{B V}
\end{aligned}
$$

[Equation was amended.] where $k_{0}$ is the electrochemical rate constant, $\alpha$ and $\beta$ are the transfer coefficients ${ }^{19 ; 20}, E$ is the electrode potential, $E_{f}^{0}$ is the formal potential of the reaction (5), and all other variables have their usual significance. At the cylinder surface we implement adsorption and desorption processes*:

$$
\begin{aligned}
\left.D \partial_{r, z} c_{B}(\vec{r}, t)\right|_{\vec{r} \in \mathbb{S}} & =k_{a} c_{B}(\vec{r}, t)\left(\Gamma_{B}^{*}-\Gamma_{B}(\vec{r}, t)\right)-k_{d} \Gamma_{B}(\vec{r}, t) \\
& =f_{\text {ads }}
\end{aligned}
$$

[Equation was amended.] where $\Gamma_{B}(\vec{r}, t)$ is the surface concentration of adsorbed species $B, \Gamma_{B}^{*}$ is the maximum surface concentration that can be reached, and $\mathbb{S}$ is the cylinder surface. It is noted that the chosen adsortion model (8) satisfies Langmuir isotherm at equilibrium or for sufficiently long conditioning times and if mass transport is solely due to diffusion processes.

\subsection{Computational methods}

The equations outlined in Section 2.1 are solved via the finite difference method, while mass transport equations are evaluated via the Alternating Differences Implicit (ADI) method and ad- and desorption processes are calculated explicitly and separately in between individual time steps. The so-obtained matrix equations are solved through the tri-diagonal matrix algorithm or 'Thomas algorithm'. As similar numerical methods are commonly used in electrochemical studies ${ }^{15 ; 21 ; 22}$ and the exact numerical approach is of no particular interest in the following, we refer the interested reader to standard textbooks including ${ }^{10}$, Chapter 9 , which provides a detailed explanation of the ADI method alongside example code.

The simulation is implemented in $\mathrm{C}++$ and makes use of the OpenMP Application Programming Interface (API) for parallel computing. Further data processing is done in Python and employs the packages NumPy and matplotlib for data processing and visualisation.

As pointed out in previous work ${ }^{23}$ software testing and convergence studies are crucial and mandatory elements of the development of numerical simulations and were carried out thoroughly along the lines of a preceding study ${ }^{22}$.

*We note that in contrast to all of the above boundary conditions, the employed adsorption process (8) does not fulfil the symmetry condition (6). Since this work however solely focuses on the height of the first voltammetric peak in a single forward scan, presented results are a good approximation of the actual processes at a reasonable computational expense. For the modelling of an entire voltammogram diffusion equation has to be solved for all relevant species, which would be an exciting topic in a future study. 


\begin{tabular}{ll} 
Parameter & Dimensionless definition \\
\hline Radial distance & $R=\frac{r}{r_{e}}$ \\
Axial distance & $Z=\frac{z}{r_{e}}$ \\
Time & $\begin{array}{l}D_{B} t \\
r_{e}^{2}\end{array}$ \\
Potential & $\theta=\frac{F}{R_{u} T}\left(E-E_{f}^{0}\right)$ \\
Current & $J=\frac{I}{2 \pi F r_{e} D_{B} c_{B}^{*}}$ \\
Concentration & $C_{j}=\frac{c_{j}^{*}}{c_{B}^{*}}$ \\
Electrochemical rate constant & $K_{0}=\frac{r_{e} k_{0}}{D_{B}}$ \\
Adsorption rate & $K_{a}=\frac{r_{e}^{2} c^{*}}{D_{B}} k_{a}$ \\
Desorption rate & $K_{d}=\frac{r_{e}^{2}}{D} k_{d}$ \\
Surface concentration & $\sigma=\frac{r_{e}^{2} F v}{D_{B} R_{u} T}$ \\
Scan rate &
\end{tabular}

Table 1: Conversions of dimensional quantities into their dimensionless equivalents based on commonlyused textbook ${ }^{10}$ definitions. $v$ represents the scan rate and $R_{u}$ is the universal gas constant.

\begin{tabular}{lll} 
Parameter & Symbol & Value \\
\hline Cylinder radius & $R_{e}$ & 1 \\
Cylinder height & $Z_{e}$ & 100 \\
Radius of the diffusion domain & $R_{\max }$ & 1.05 \\
Electrochemical rate constant & $K_{0}$ & 10 \\
Transfer coefficient ${ }^{19 ; 20}$ & $\alpha$ & 0.5 \\
Adsorption rate & $K_{a}$ & $10^{-3}$
\end{tabular}

Table 2: List of the dimensionless parameter values used in all simulations if not otherwise specified.

\subsection{Dimensionless coordinates}

For a more convenient application of our results to various experimental conditions, data is presented in the dimensionless coordinates defined in Table 1. Table 2 further lists parameter values used in the below simulations if not specified otherwise.

\section{Results and discussion}

This section commences with a comparison of example voltammograms to illustrate the impact of adsorption processes on the observed current response. We then progress to investigate the dependency of the peak current response on various parameters before we finally discuss the design of future experiments in the light of our theoretical findings. 


\subsection{Influence of adsorption on the forward scan}

We start our analysis with exemplary voltammograms that illustrate the influence of adsorption on the current response in the forward scan. Figure 3 compares data obtained for the cases of no adsorption and that adsorption can take place at the cylinder surface. In the latter, the initial surface concentration is set according to Langmuir isotherm, which in effect assumes the experiment is performed after a sufficient conditioning time to allow equilibrium between the surface and the solution. Figure 3 clearly demonstrates that in the same geometry adsorption along the lines of Equation (8) can lead to a significant amplification of the current signal, which for the given set of parameters is increased by ca ten times greater than the signal in the case of no adsorption. This observation can be understood as follows: If adsorption is enabled and after a sufficient conditioning time, the system will approach the Langmuir equilibrium prior to the first scan. The analyte material $B$ is hence pre-concentrated at the cylinder surface leading to a much greater number of analyte molecules being located in the immediate proximity of the electrode. Once the forward scan has been triggered, analyte in the solution near the electrode will be consumed, shifting the system locally out of the Langmuir equilibrium. The absolute value of the second term in Equation (8) will then exceed the value of the first and analyte is released from the cylinder into the solution near the electrode, elevating the concentration in comparison to the case of no adsorption and enabling the observed enhanced voltammetric response. Importantly, different ratios of the adsorption- and desorption rates $K_{a}$ and $K_{d}$ lead to significant differences in the amplification observed at the same scan rate: At a fixed $K_{a}$, fast desorption cause a quick release of adsorbed material at the expense of less material being pre-concentrated in the first place, while slow desorption rates preconcentrate a large amount of material at the electrode but only slowly release it into the solution where it is detected. As a result we find a maximal amplification at an intermediate $K_{a}$.

The effect is further illustrated in Figure 4, which shows the temporal evolution of the surface coverage of adsorbed species as a function of their position on the cylinder and the time. Here, it can be clearly seen that, first, the amount of pre-concentrated material varies for different desorption rates and, second, once material in solution is consumed by the electrode, the area affected by changes in the surface coverage increases at different rates as material is released at different rates. In all three cases, the latter effect initially only affects the cylinder surface in the immediate proximity of the electrode but then continues to occur in greater distance as the electrode diffusion field embraces larger volumes in solution.

\subsection{Amplification of the peak current}

Having shown that adsorption- and desorption processes in porous electrodes may aid the detection of an analyte under some conditions, we progress by identifying what more exactly these conditions are. To this end, we investigate the height of the peak during the forward scan as a function of the ratio:

$$
K_{a d s}=\frac{K_{a}}{K_{d}}
$$

and for various scan rates while $K_{d}$ is varied and all other parameters are held constant. Figure 5a depicts the amplification factor, namely the peak height divided by the peak height found under the same experimental conditions but without adsorption, for different adsorption rates $K_{d}$ and three different scan rates. The analysis reveals that the amplification factor can reach values of up to ca ten, while the optimal $K_{a d s}$ depends on the chosen scan rate. We further note that for both high and low $K_{a d s}$ the amplification 


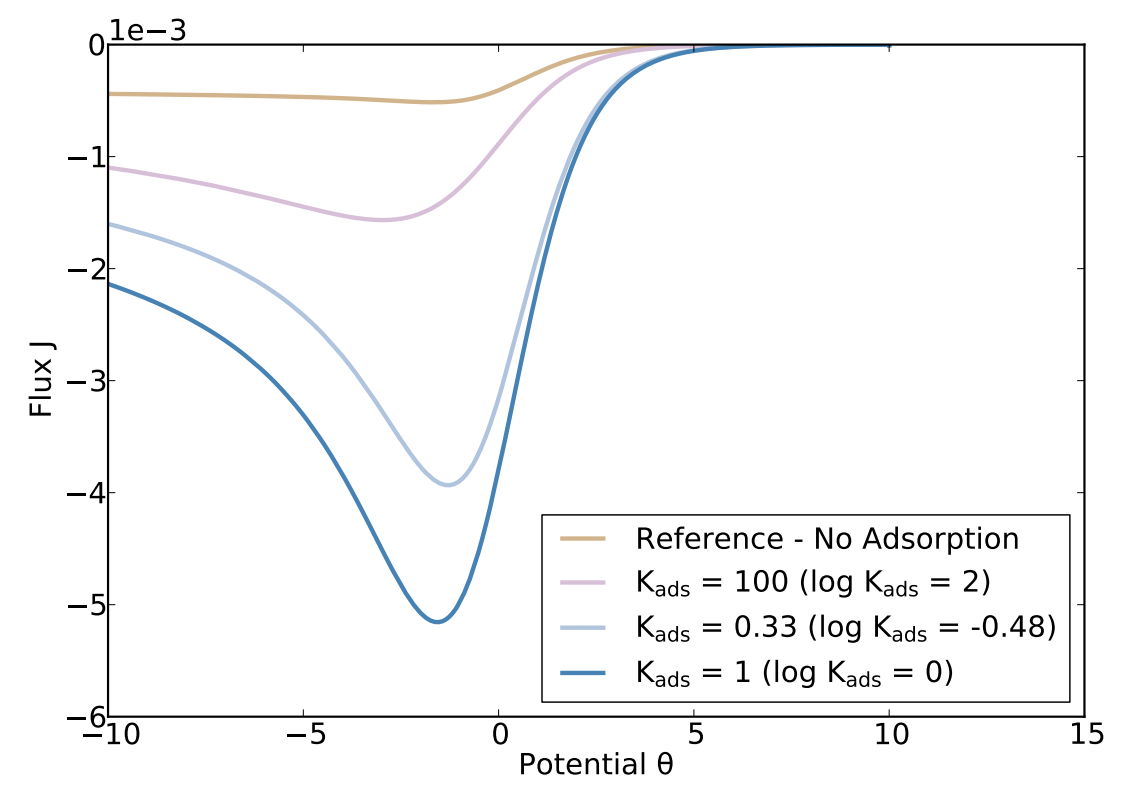

Figure 3: Voltammetric electrode response during the forward scan for the cases of no adsorption and adsorption via Equation (8). In the latter case, the initial surface concentration satisfies Langmuir isotherm.

factor tends to one since either the rate of material being released is negligible as adsorption dominates or no material is initially pre-concentrated within the porous layer. It is further noted that with an increasing scan rate, the optimal peak shifts to faster $K_{d}$, i.e. to lower $K_{a d s}$, which we address in the following paragraph. Importantly, adsorption processes along the lines of Equation (8) never reduce the current response in the here-investigated model.

Figure $5 \mathrm{~b}$ describes the amplification factor as a function of the scan rate for two different geometries, where the cylinder height at a fixed cylinder radius is varied. For the case of a radius-to-height ratio of 1:100 (blue dots), we find that the amplification factor passes a maximum at an optimal scan rate, which is due to the interplay between the amount of pre-concentrated material being released and its release rate: At slow scan rates, the desorption rate is small as the ratio of ad- and desorbed material at the cylinder surface is always near equilibrium and the release of material triggered by the locally unbalanced Langmuir equilibrium is hence slow as well. This slow release rate in combination with the fact the the overall amount of material initially adsorbed is the same for all scan rates leads to a slower rate of initially adsorbed material reacting at the electrode. In contrast, most of the adsorbed material does not desorb at fast scan rates as the diffusion field does not extend far enough to trigger its release. The rate at which the affected small amount of material desorbes is however then comparably fast.

Interestingly, the direct comparison between the 1:100 and 1:10 geometries in Figure 5b further reveals that the peak amplification factors appear at different scan rates. The scan rate at which the highest amplification in the 1:10 geometry is observed exceeds the one of the 1:100 geometry by more than one order of magnitude, while both geometries converge to an identical response for large scan rates. This observation can be understood by considering the extent to which the diffusion field expands. In the 1:10 geometry, the diffusion field simulated for the instant of the peak current and the highest amplification 


$$
K_{a d s}=100
$$

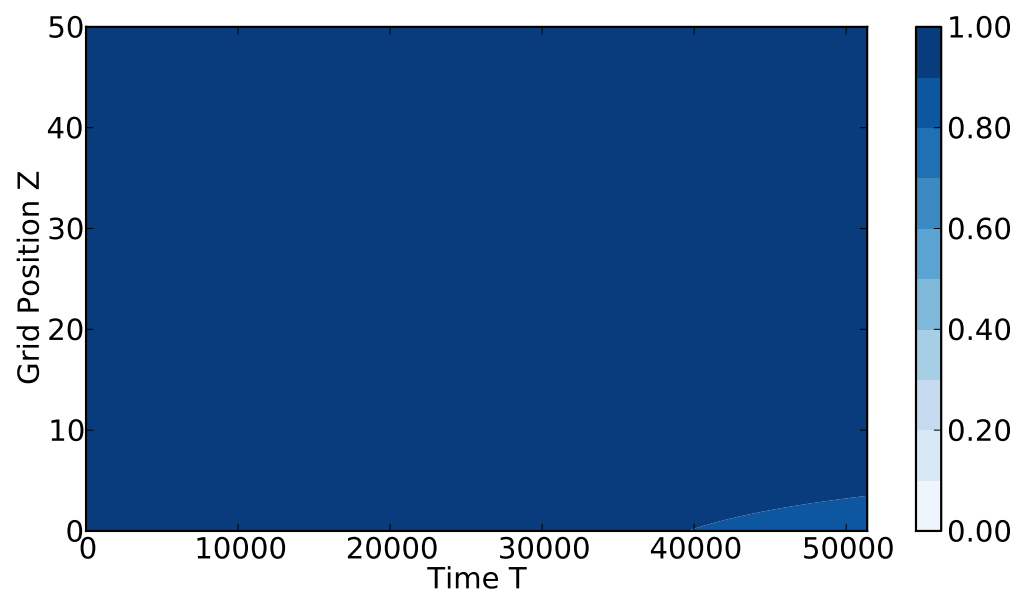

$$
K_{a d s}=1
$$

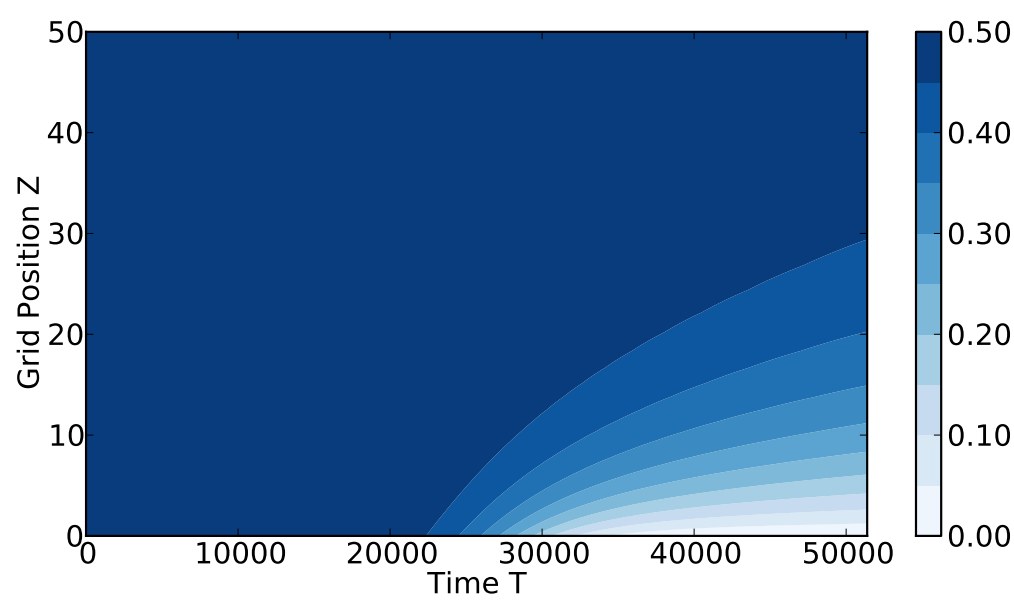

$$
K_{a d s}=0.33
$$

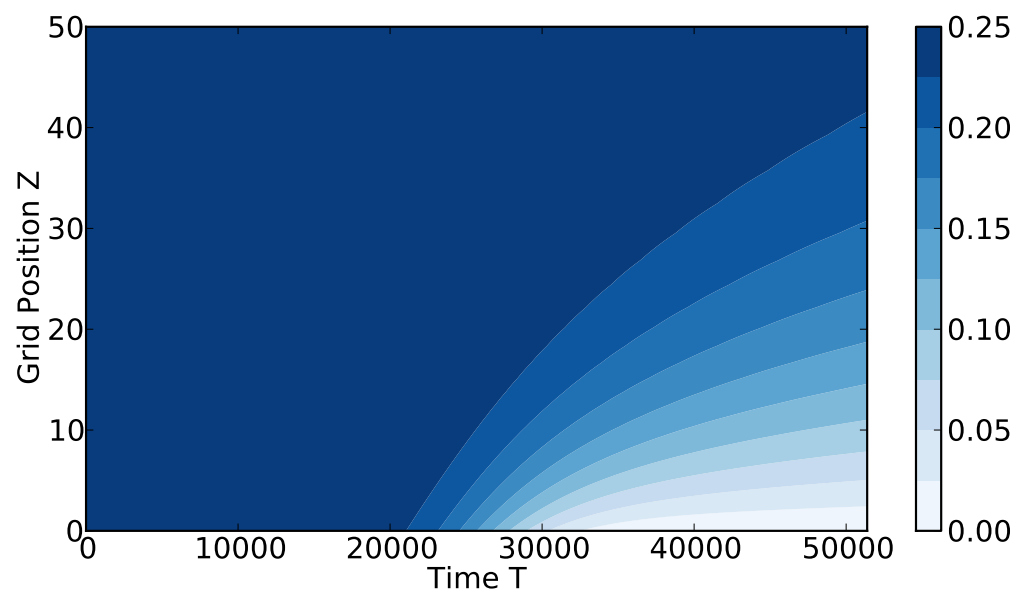

Figure 4: [Figure was amended.] Surface coverage $\theta_{B}=\Gamma_{B} / \Gamma_{B}^{*}$ of adsorbed analyte molecules $B$ on the cylinder surface during the forward scans in Figure 3. The y-axis depicts the Z-position of the presented $\theta_{B}$, i.e. the Z-position with increasing distance from the electrode, while the x-axis shows the dimensionless time. It is noted that we plot the the range $R \in[0,50]$ of a cylinder featuring the length 100 and vary the color coding for a clearer illustration. 


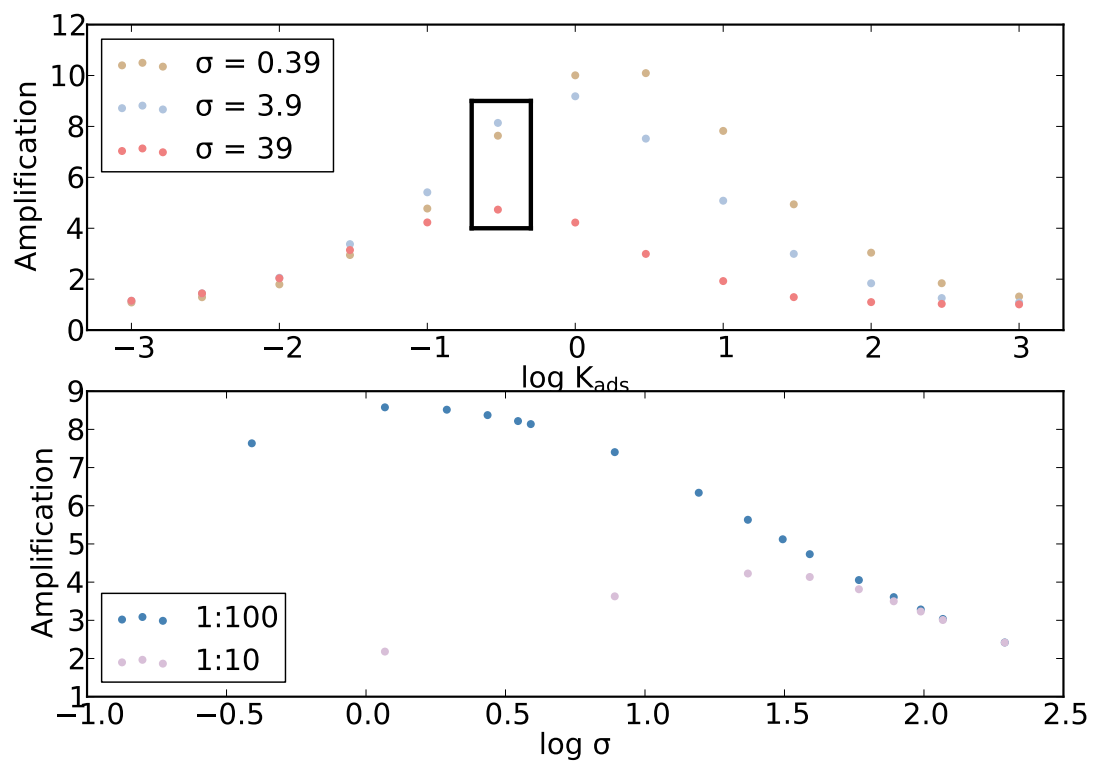

Figure 5: Amplification factor of the height of the first peak if compared with the same experiment without adsorption. a) Amplification factor as a function of the ratio of the adsorption and desorption rate $K_{a d s}=K_{a} / K_{d}$ calculated for three different scan rates and at a fixed $K_{a}$. b) More detailed study of the points highlighted in a) with respect to the scan rate. In addition to the geometry considered in a) the same analysis is carried out for a cylinder featuring a height-to-radius ratio of 10:1 rather than 100:1. The logarithmic scaling of the $\mathrm{x}$-axis is calculated with respect to the base ten.

factor embraces about the entire cylinder as shown in Figure 6a might be intuitively anticipated. At slower scan rates, where the diffusion field expands even further, no advantage can hence be gained as the pre-concentrated material is consumed and the amplification is lowered due to the slower release of adsorbed material. The optimal scan rate is therefore determined by the height of the cylinder. At faster scan rates, no difference can be seen between the 1:10 and 1:100 geometry as the diffusion field does not reach areas in which the two geometries differ. In stark contrast, the diffusion field of the 1:100 geometry depicted in Figure 6b does not cover the entire cylinder height, but solely the lower part of the cylinder. The optimal scan rate is hence solely determined by the above-explained interplay between the rate of material desorbing and the amount of adsorbed material affected by the electrode diffusion field, and greater cylinder heights would exhibit the same voltammetric response.

For a more intuitive illustration of the results, we additionally calculate the results shown in Figure 5 on the basis of the representative set of dimensional parameters shown in Table 3. The data clearly shows that peak amplification lies well in the experimentally accessible range of scan rates.

\subsection{Implications on the design of experiments}

The above discussion revealed that adsorption inevitably affects the performance of a sensor and has direct implications to the design of experiments. While parameters such as the adsorption- and desorption rate, $k_{a}$ and $k_{d}$, are not adjustable and entirely beyond the influence of the experimentalist, the scan rate can be set precisely. Though amplification factors are of course dependent on the geometry of the electrode 

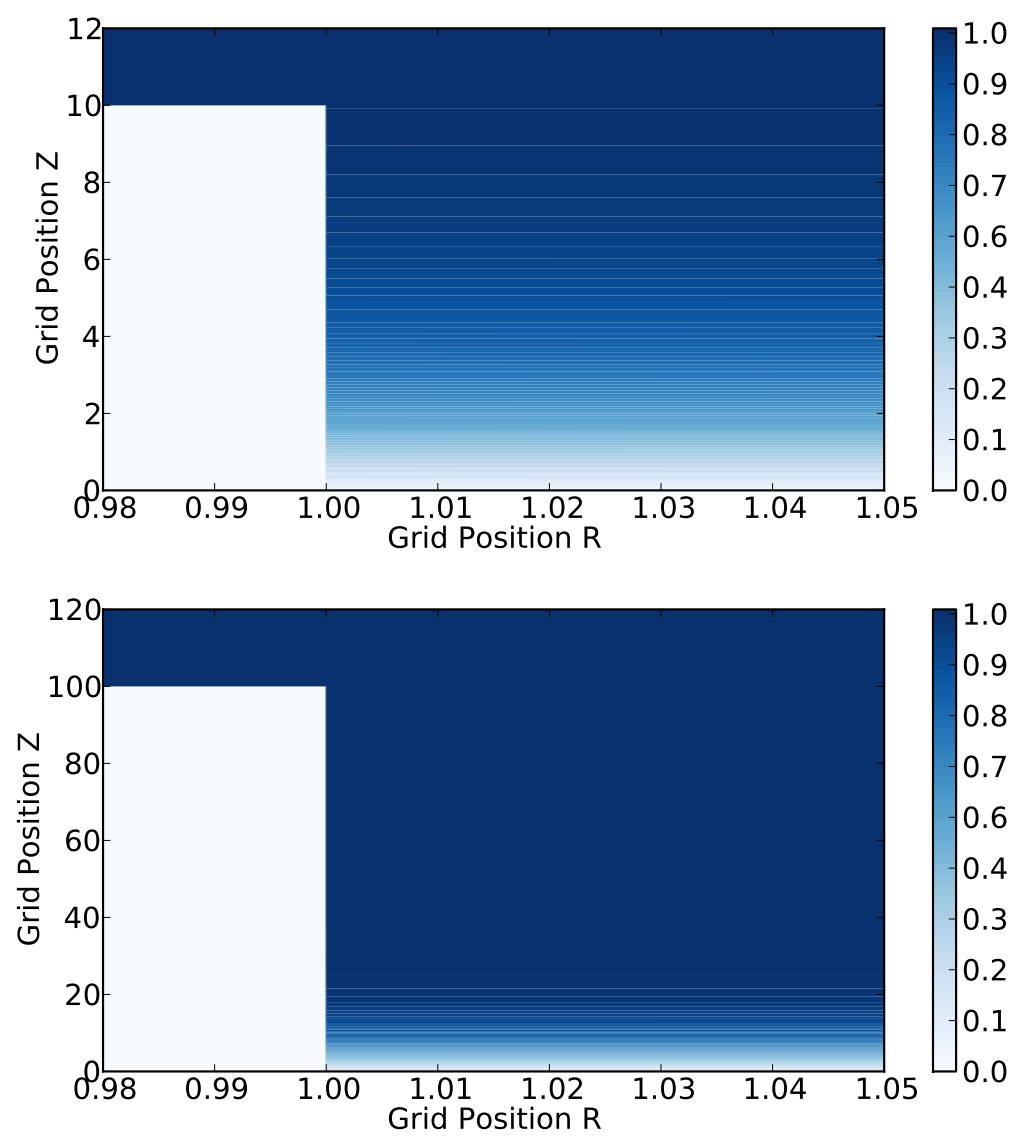

Figure 6: Concentration profiles simulated for the highest amplification factors observed in Figure 5b at the moment of the peak current. a) and b) depict the 1:10 and 1:100 geometries, respectively.

\begin{tabular}{|c|c|c|}
\hline Parameter & Symbol & Value \\
\hline Cylinder radius & $r_{e}$ & $1 \mu \mathrm{m}$ \\
\hline Cylinder height & $h_{e}$ & 10 or $100 \mu \mathrm{m}$ \\
\hline Radius of the diffusion domain & $r_{\max }$ & $1.05 \mu \mathrm{m}$ \\
\hline Electrochemical rate constant & $k_{0}$ & $0.01 \mathrm{~m} \mathrm{~s}^{-1}$ \\
\hline Bulk concentration & $c_{B}^{*}$ & $1 \mathrm{mM}$ \\
\hline Diffusion coefficient & $D_{A}=D_{B}$ & $10^{-9} \mathrm{~m}^{2} \mathrm{~s}^{-1}$ \\
\hline Transfer coefficient ${ }^{19 ; 20}$ & $\alpha$ & 0.5 \\
\hline Adsorption rate & $k_{a}$ & $1 \mathrm{~m}^{3} \mathrm{~s}^{-1} \mathrm{~mol}^{-1}$ \\
\hline Maximum surface concentration & $\Gamma_{B}^{*}$ & $1.67 \cdot 10^{-9} \mathrm{~mol} \mathrm{~m}^{-2}$ \\
\hline
\end{tabular}

Table 3: List of the dimensioned parameter values used in all simulations if not otherwise specified.

as well as on $k_{a}$ and $k_{d}$, the amplification necessarily passes a maximum if the scan rate is varied. A study of the amplification factor as a function of the scan rate, which for instance could be a simple plot of $I_{p}$ vs $\sqrt{v}$, hence provides vital insight into how the optimal scan rate should be set.

In spite of the attractive amplification factors resulting from the pre-concentration of analyte within the porous layer, it is nonetheless crucial to compare the performance of a porous electrode system with a simple planar electrode of the same size when designing experimental set-ups for analytical applications. ${ }^{24}$ As the decoration of an electrode with an insulating but adsorbing porous layer necessarily leads to parts 

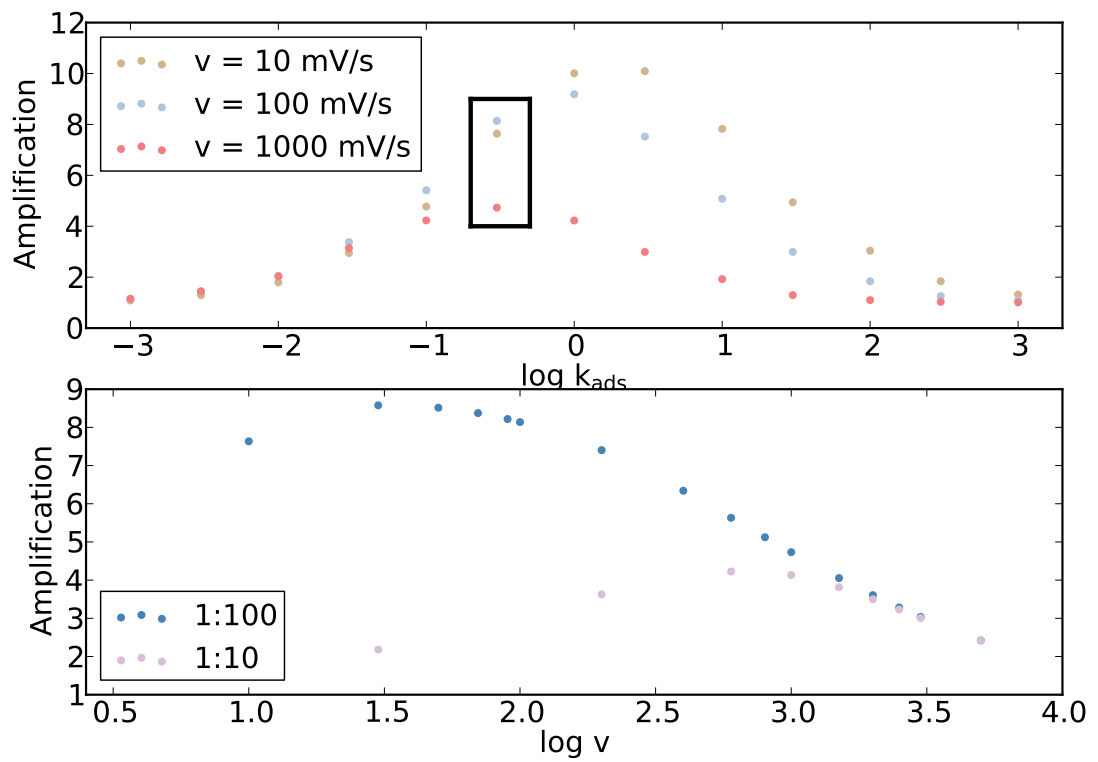

Figure 7: Dimensional plot of the data shown in Figure 5 calculated on the basis of the parameters shown in Table 3. The logarithmic scaling of the x-axis is calculated with respect to the base ten and $K_{a d s}$ is provided in units of $\mathrm{m}^{3}$.

of the electrode being blocked and hence being unavailable for the detection of analyte, depending on the geometry, the advantage gained from the porous layer may not outbalance the inevitable associated loss of signal. For the given 1:100 electrode geometry, a related estimate can be made from the Randles-Ševčík equation. For an unblocked electrode we know that:

$$
I_{p}=0.446 F A c_{i}^{*} \sqrt{\frac{F D_{i} v}{R_{u} T}}
$$

where $A$ is the active electrode surface area. Given that the above equation describes the response of a macroelectrode, it is immediately clear that the same equation is directly applicable to the here-considered porous electrode if the scan rate is sufficiently fast to enable one-dimensional diffusion within the pore. In this case, one can exploit (10) to estimate the ratio of the peak heights found for a porous- and a planar electrode to:

$$
\frac{I_{p}(\text { porous })}{I_{p}(\text { planar })}=\frac{f_{A} A(\text { porous })}{A(\text { planar })}=\frac{f_{A} \pi\left(r_{\max }^{2}-r_{e}^{2}\right)}{\pi r_{\max }^{2}}
$$

where $f_{A}$ is the corresponding amplification factor, $A$ (porous) the entire active surface area of the porous electrode, i.e. $n \pi\left(r_{\text {max }}^{2}-r_{e}^{2}\right)$ with $n$ being the number of diffusion domains, and $A($ planar $)$ is the surface area of the planar electrode. Interestingly for the above 1:100 example the ratio (11) yields a value of 0.93 revealing that a planar electrode would actually exhibit a slightly better performance. If, unlike here, the pores are not deep enough to allow the one-dimensional diffusion approximation or the geometry is more complex, absolute peak values can nonethless directly be compared with Randles-Ševčík equation (10) to determine whether a planar electrode is more effective. 


\section{Conclusions}

We demonstrate that adsorption- and desorption processes in porous materials can aid the electrochemical detection of an analyte. The advantage is due to the pre-concentration of adsorbed analyte within the porous material and its subsequent fast release as soon as the consumption of electro-active species at the electrode unbalances the Langmuir equilibrium. For the investigated system, we found amplification factors of up to ten if compared to the case without adsorption, depending on the geometry, the scan rate, and prevalent adsorption- and desorption processes.

While the consideration of adsorption- and desorption processes can be highly beneficial in the design of future experiments, we though note that under certain circumstances (including the case of the hereinvestigated geometries) a planar macroelectrode may be superior to a porous electrode: As the porous layer effectively blocks part of the underlying electrode surface, the large amplification factors found with respect to non-adsorbing porous electrodes do not necessarily guaranty a better signal than a simple flat electrode surface. It is therefore generally helpful to compare the performance of porous electrodes with the value predicted by Randles-Ševčík equation to evaluate whether the possibly invested effort pays off. Further it can be stressed that the amplification realised is unlikely to exceed one order of magnitude unless multi-layer adsorption prevails and, further, given the complex interplay between adsorption cause and rates the a priori design of porous sensors is challenging.

\section{Acknowledgements}

The research leading to these results has received partial funding from the European Research Council under the European Union's Seventh Framework Programme (FP/2007-2013) / ERC Grand Agreement n. $[320403]$.

\section{References}

[1] I.Streeter, G. G. Wildgoose, L. Shao, and R. G. Compton. Cyclic voltammetry on electrode surfaces covered with porous layers: An analysis of electron transfer kinetics at single-walled carbon nanotube modified electrodes. Sensors and Actuators B: Chemical, 133(2):462-466, 2008.

[2] M. J. Sims, N. V. Rees, E. J. F. Dickinson, and R. G. Compton. Effects of thin-layer diffusion in the electrochemical detection of nicotine on basal plane pyrolytic graphite (BPPG) electrodes modified with layers of multi-walled carbon nanotubes (MWCNT-BPPG). Sensors and Actuators B: Chemical, 144(1):153-158, 2010.

[3] M. C. Henstridge, E. J. F. Dickinson, M. Aslanoglu, C. Batchelor-McAuley, and R. G. Compton. Voltammetric selectivity conferred by the modification of electrodes using conductive porous layers or films: The oxidation of dopamine on glassy carbon electrodes modified with multiwalled carbon nanotubes. Sensors and Actuators B: Chemical, 145(1):417-427, 2010.

[4] X. Bo, M. Zhou, and L. Guo. Electrochemical sensors and biosensors based on less aggregated graphene. Biosensors and Bioelectronics, 89:167-186, 2017. 
[5] Z-H Huang, Z. Yang, F. Kang, and M. Inagaki. Carbon electrodes for capacitive deionization. J. Mater. Chem. A, 5(2):470-496, 2017.

[6] J. Wang, P. Nie, B. Ding, S. Dong, X. Hao, H. Dou, and X. Zhang. Biomass derived carbon for energy storage devices. J. Mater. Chem. A, 5(6):2411-2428, 2017.

[7] J. Friedl and U. Stimming. Determining electron transfer kinetics at porous electrodes. Electrochimica Acta, 227:235-245, 2017.

[8] M. D. Levi, N. Shpigel, S. Sigalov, V. Dargel, L. Daikhin, and D. Aurbach. In situ porous structure characterization of electrodes for energy storage and conversion by EQCM-d: a review. Electrochimica Acta, 232:271-284, 2017.

[9] R. G. Compton and C. E. Banks. Understanding Voltammetry, 2nd Edition. Imperial College Press, 2011.

[10] R. G. Compton, E. Laborda, and K. R. Ward. Understanding voltammetry: Simulation of electrode processes. Imperial College Press, 2013.

[11] B. R. Scharifker. Diffusion to ensembles of microelectrodes. Journal of Electroanalytical Chemistry and Interfacial Electrochemistry, 240(1-2):61-76, 1988.

[12] B. A. Brookes, T. J. Davies, A. C. Fisher, R. G. Evans, S. J. Wilkins, K. Yunus, J. D. Wadhawan, and R. G. Compton. Computational and experimental study of the cyclic voltammetry response of partially blocked electrodes. part 1. nonoverlapping, uniformly distributed blocking systems. The Journal of Physical Chemistry B, 107(7):1616-1627, 2003.

[13] T. J. Davies, C. E. Banks, and R. G. Compton. Voltammetry at spatially heterogeneous electrodes. Journal of Solid State Electrochemistry, 9(12):797-808, 2005.

[14] N. Godino, X. Borrisé, F. X. Muñoz, F. Javier del Campo, and R.G. Compton. Mass transport to nanoelectrode arrays and limitations of the diffusion domain approach: Theory and experiment. The Journal of Physical Chemistry C, 113(25):11119-11125, 2009.

[15] M. C. Henstridge, E. J. F. Dickinson, and R. G. Compton. Mass transport to and within porous electrodes. linear sweep voltammetry and the effects of pore size: The prediction of double peaks for a single electrode process. Russian Journal of Electrochemistry, 48(6):629-635, 2012.

[16] O. Sliusarenko, A. Oleinick, I. Svir, and C. Amatore. Validating a central approximation in theories of regular electrode electrochemical arrays of various common geometries. Electroanalysis, 27(4):980$991,2015$.

[17] J. K. Novev, S. Eloul, and R. G. Compton. Influence of reaction-induced thermal convection on the electrical currents measured in chronoamperometry and cyclic voltammetry. The Journal of Physical Chemistry C, 120(25):13549-13562, 2016.

[18] E. J. F. Dickinson, J. G. Limon-Petersen, N. V. Rees, and R. G. Compton. How much supporting electrolyte is required to make a cyclic voltammetry experiment quantitatively "diffusional"? a theoretical and experimental investigation. The Journal of Physical Chemistry C, 113(25):11157$11171,2009$. 
[19] R. Guidelli, R. G. Compton, J. M. Feliu, E. Gileadi, J. Lipkowski, W. Schmickler, and S. Trasatti. Defining the transfer coefficient in electrochemistry: An assessment (IUPAC technical report). Pure and Applied Chemistry, 86(2):245-258, 2014.

[20] R. Guidelli, R. G. Compton, J. M. Feliu, E. Gileadi, J. Lipkowski, W. Schmickler, and S. Trasatti. Definition of the transfer coefficient in electrochemistry (IUPAC recommendations 2014). Pure and Applied Chemistry, 86(2):259-262, 2014.

[21] Z. Ban, E. Kätelhön, and R. G. Compton. Voltammetry of porous layers: Staircase vs analog voltammetry. Journal of Electroanalytical Chemistry, 776:25-33, 2016.

[22] H. T. H. Chan, E. Kätelhön, and R. G. Compton. Voltammetry using multiple cycles: Porous electrodes. To be published.

[23] E. Kätelhön and R. G. Compton. Testing and validating electroanalytical simulations. The Analyst, 140(8):2592-2598, 2015.

[24] E. Kätelhön. Theory in modern electrochemistry: Science for the academic ivory tower or a vital tool for today's research? Research and Reviews in Electrochemistry, 8(1):E1-E2, 2017. 